Rabaska

Revue d'ethnologie de l'Amérique française

\title{
Mémoires et thèses
}

\section{Jean-Pierre Pichette}

Numéro 1, 2003

URI : https://id.erudit.org/iderudit/201632ar

DOI : https://doi.org/10.7202/201632ar

Aller au sommaire du numéro

Éditeur(s)

Société québécoise d'ethnologie

ISSN

1703-7433 (imprimé)

1916-7350 (numérique)

Découvrir la revue

Citer ce document

Pichette, J.-P. (2003). Mémoires et thèses. Rabaska, (1), 203-210.

https://doi.org/10.7202/201632ar

Ce document est protégé par la loi sur le droit d'auteur. L'utilisation des services d'Érudit (y compris la reproduction) est assujettie à sa politique d'utilisation que vous pouvez consulter en ligne.

https://apropos.erudit.org/fr/usagers/politique-dutilisation/
Cet article est diffusé et préservé par Érudit.

Érudit est un consortium interuniversitaire sans but lucratif composé de l'Université de Montréal, l'Université Laval et l'Université du Québec à Montréal. Il a pour mission la promotion et la valorisation de la recherche. https://www.erudit.org/fr/ 


\section{Annuel}

\section{Mémoires et thèses}

\section{Jean-Pierre Pichette}

Université de Sudbury

Nous publions ici les résumés des mémoires et des thèses déposés entre 1998 et 2000 et venus à notre connaissance : seize proviennent de l'université Laval et un de l'Université d'Ottawa. Merci à Mme Suzanne Allaire, de la faculté des Études supérieures de l'université Laval, qui nous a communiqué la liste des étudiants diplômés de son institution.

Benjamin, ViRginie. Une ferme à vocation arboricole: la Ferme des Coteaux de Saint-Joachim, 1850-1900. Mémoire (M.A.), Université Laval, 1999, [vi]136 p. ill. [Direction : Laurier Turgeon].

La Ferme des Coteaux, située dans la paroisse de Saint-Joachim, sur la côte de Beaupré, a été créée en 1818 par le Séminaire de Québec ; elle en est toujours sa propriété. À partir d'archives manuscrites, comme des rapports d'inspection et les livres de comptes, ce mémoire explore le passé de cette ferme institutionnelle, en s'attardant particulièrement aux années 1850 à 1900. Pendant cette période, c'est le fermier Hugh Brown, un immigrant écossais, et sa famille, qui assument la production à la Ferme des Coteaux. Après avoir décrit le contexte général de l'agriculture dans la seconde moitié du XIX ${ }^{\mathrm{e}}$ siècle, nous présentons la situation de l'agriculture sur la côte de Beaupré ainsi qu'un bref historique des activités agricoles du Séminaire de Québec. La seconde partie du mémoire s'attarde à la Ferme des Coteaux elle-même, c'est-à-dire à son histoire, à ses habitants, ainsi qu'à sa culture matérielle. Enfin, la troisième partie, le cœur même du mémoire, explore les différentes productions de la ferme : les animaux, les céréales, les légumes et, surtout, l'arboriculture fruitière.

Fillion, PASCAL. Étude de l'univers domestique en milieu bourgeois chez les anglophones et les francophones du Québec : le cas Jourdain-Fiset. Mémoire (M. A.), Université Laval, 1998, ix-116 p. ill., cartes. [Direction : Jocelyne Mathieu].

Notre étude porte sur le fonds Jourdain-Fiset, acquis en 1993 par le Musée de la civilisation, qui représente la presque totalité d'un univers domestique dans lequel ont évolué cinq générations de la famille Tourangeau-Fiset aux XIX ${ }^{e}$ et $\mathrm{XX}^{\mathrm{e}}$ siècles. Son caractère exceptionnel 
découle surtout du fait que les objets qui composent le fonds représentent des ensembles constitués qui sont, pour la grande majorité, en parfait état de conservation. L'objectif de notre étude est de découvrir l'existence éventuelle d'un modèle d'organisation spécifique de la vie domestique de la famille Tourangeau-Fiset. L'analyse du fonds devait permettre de dégager des éléments permettant d'affirmer que l'organisation domestique suivait un modèle semblable à ce que l'on retrouve dans les maisons bourgeoises anglophones.

Gadoury, Marc. Sir Henri Gustave Joly de Lotbinière: visionnaire et promoteur de la conservation des forêts, au Québec, à la fin du XIX ${ }^{\mathrm{e}}$ siècle. Mémoire (M. A.), Université Laval, 1998, iv-117 p. ill. [Direction : Marcel Moussette ; codirection : Jean-Claude Dupont].

Figure importante de l'histoire environnementale au Québec, Sir Henri Gustave Joly de Lotbinière s'est partagé, toute sa vie durant, entre une carrière politique remarquable et sa véritable passion : la foresterie. À la fin du XIX ${ }^{\mathrm{e}}$ siècle, grâce à son implication active dans le mouvement de conservation des forêts, il a suscité auprès des gouvernements et de la population, un intérêt pour la conservation et la préservation des ressources forestières. Visionnaire, il l'a été en réprouvant l'exploitation effrenée du couvert forestier et en remettant en question le mythe de la « forêt inépuisable ». Promoteur, il l'a été aussi en donnant la première impulsion au mouvement de conservation de la forêt sur le territoire. Cette recherche explore le rôle de Sir Henri Gustave Joly de Lotbinière dans le mouvement de conservation au Québec, dès la fin du XIX ${ }^{e}$ siècle, et veut démontrer que, sous son impulsion et son exemple, une prise de conscience s'est engagée, un embryon de réflexion s'est constitué appelant un nouveau rapport de l'homme à la forêt sur le territoire.

GaGnE-Collard, Agathe. La Consommation vestimentaire à Québec, 1940 1990 : le cas du pantalon féminin. Thèse (Ph. D.), Université Laval, 2000, 219 p. ill. [Direction : Jocelyne Mathieu ; codirection : Pierre Beaudoin].

Le vêtement joue sa partie aux différentes étapes de la vie, de la naissance à la mort. Avec l'entrée en scène d'une consommation vestimentaire effrénée, depuis la dernière guerre, le port du pantalon devient le reflet d'un désir d'émancipation. D'abord utilitaire dans un cadre occasionnel de travail, le pantalon a pris sa place, progressivement, dans la garderobe féminine. Timidement d'abord, il est devenu expression d'un quotidien où la femme joue un nouveau rôle. Des matériaux, des modèles et des couleurs témoignent de l'importance des modes dans un système incontournable de consommation. Longtemps, le couple a été divisé par la forme des vêtements tant dans la nature des activités que dans les 
responsabilités. Cette mutation des gestes séculaires, ce changement de trajectoire du dimorphisme sexuel vestimentaire est manifeste dans le monde occidental. Les sources d'information iconiques, scripto-iconiques et ethnographiques nous révèlent un vêtement porteur de messages au regard d'une coutume où des hésitations et des résistances font place aux innovations.

GIRARD, DENISE. Différenciation sociale et rituels du mariage : les Montréalais francophones, 1925-1940. Thèse (Ph. D.), Université Laval, 1998, x-295 p. ill. [Direction : Anne-Marie Desdouits]. [Thèse publiée sous le titre Mariage et classes sociales. Les Montréalais francophones entre les deux Guerres, Sainte-Foy, Les Éditions de l'IQRC [et les PUL], « Culture et société », 2000, $203 \mathrm{p}$. Voir le compte rendu dans ce numéro].

Les rituels de mariage sont utilisés comme marqueurs culturels de la différenciation sociale dans la société montréalaise francophone entre 1925 et 1940. Les données sur lesquelles s'appuie la présente étude ont été recueillies lors d'une enquête concernant trente-six mariages ayant été célébrés entre des Canadiens français, originaires de Montréal. La description ethnographique tient compte de leurs pratiques culturelles et ce, selon une division de l'échantillon en trois classes sociales: la bourgeoisie, la classe moyenne et les ouvriers. Les rituels sont ensuite analysés pour faire ressortir les différences de classes sous divers rapports : individus, familles, groupes d'âge, etc.

Hamel, Nathalie. Le Costume en Beauce, 1920-1960: tradition, innovation et régionalisme. Mémoire (M. A.), Université Laval, 1998, viii132 p. ill. [Direction : Jocelyne Mathieu].

Peut-on parler d'un costume régional beauceron ? Afin de répondre à cette question, le premier chapitre présente les rapports vêtement/tradition, en identifiant les éléments que l'on peut considérer traditionnels, d'une part dans les vêtements eux-mêmes, d'autre part dans les pratiques vestimentaires. Ces éléments sont analysés en considérant l'influence des contextes historiques et idéologiques de la période 1920-1960. Le second chapitre observe la manière dont s'intègrent les nouveautés vestimentaires dans ce contexte de survie des pratiques traditionnelles. Enfin, l'analyse de la création de costumes régionaux beaucerons et québécois, à des fins économiques et identitaires, pose la question de la place que ceux-ci occupent dans l'imaginaire collectif.

HaRvengt, David. Un bilan de la recherche au Célat : 1976-1996. Mémoire (M. A.), Université Laval, 1998, v-133 p. ill. [Direction : Laurier Turgeon].

Le CÉLAT a fêté ses vingt ans en 1996, année où le projet de cette maîtrise a été lancé. Celle-ci correspond donc à un bilan de ces années de recherche. 
Ce mémoire traite du Centre interdisciplinaire sur les lettres, les arts et les traditions dans une approche générale. Il présente le CÉLAT dans un contexte général qui a eu une influence sur le Centre, tant au niveau des instances administratives et décisionnelles qu'à celui des courants théoriques. Au-delà de la présentation des structures du CÉLAT et de ses orientations scientifiques, notre analyse a porté aussi sur l'internationalisation du Centre, l'interdisciplinarité, les publications, ainsi que le rôle tenu par l'ethnologie tout au long de ses vingt années.

Laflamme, Josée. Femmes et aire domestique, un mode de vie: modèles, valeurs et comportements. Mémoire (M. A.), Université Laval, 1997, iv193 p. ill. [Direction : Jean Du Berger].

La maison est le lieu des premiers apprentissages de la culture et la femme y joue un grand rôle d'éducatrice. Nous étudions les différents aspects du processus de socialisation et d'éducation de la femme dans l'aire domestique. Dans un contexte culturel où la seule fonction sociale était celle du mariage et de la maternité, nous voyons que chaque action développait chez la fillette et l'adolescente une attitude faite de dévouement et même du sacrifice de sa propre vie pour les siens. Le cadre des pratiques culturelles et des fonctions urbaines nous permet de décrire, à partir des témoignages que nous avons recueillis, la formation de la femme puis sa façon d'assumer par la suite les responsabilités qui étaient siennes dans le domaine domestique. Ce qui nous conduit à examiner les modèles que toutes les instances sociales contribuaient à mettre en place. Ainsi, nous reconstituons le parcours de vie de la femme, de la petite enfance à la vieillesse. Les sphères de la société urbaine de l'époque convergeaient vers la famille et la femme qui en constituaient le coeur.

Lafleur, Claude. La Céramique institutionnelle du dépôt de la firme A. E. Vallerand. Mémoire (M. A.), Université Laval, 1999, vii-177 p. ill., cartes. [Direction: Marcel Moussette].

La fouille archéologique, entreprise en 1991 [à Québec] aux coins des rues Dalhousie et de la Barricade, autrefois Saint-Antoine, a permis de mettre au jour une importante quantité d'objets de verre et de céramique ayant appartenu à la firme A. E. Vallerand, en affaires à cet endroit de 1883 à 1987. L'origine du très riche dépôt d'objets auquel nous nous sommes intéressée provient de l'utilisation d'un vide sanitaire localisé sous le plancher du rez-de-chaussée de l'édifice, à partir du début du commerce jusque vers les années 1930 ou 1940. Notre étude porte seulement sur une partie de la collection récupérée dans le sous-sol de la firme A. E. Vallerand, soit la céramique institutionnelle, communément 
appelée Hotel Ware, une vaisselle ornée, dans la plupart des cas, d'un logo annonçant le commerce. L'objet de cette recherche est d'étudier cette céramique, les logos et les devises qui y sont inscrits, ainsi que les manufactures et les agents de manufacturiers en faisant le commerce afin de mieux la connaître et la relier au milieu social où elle prit naissance.

LAFONTAINE, NANCY. L'Iconographie historique et ouvrière d'Ozias Leduc à Shawinigan-Sud. Mémoire (M. A.), Université Laval, 1999, 133 p. [Direction : Jean Simard].

Ozias Leduc a exécuté quinze tableaux dans l'église Notre-Dame-de-laPrésentation à Shawinigan-Sud. Six illustrent la découverte de la ville et les travaux traditionnels des Shawiniganais, ce qui est peu commun dans un lieu de culte. L'illustration de la vie des travailleurs a-t-elle suscité chez les Sud-Shawiniganais un sentiment d'appartenance à leur église? Ce mémoire vise donc à découvrir si les peintures représentant l'histoire et la profession, que pratiquent encore de nombreux citoyens, ont trouvé écho dans la population ou si elles n'intéressent que les amateurs d'art. C'est donc l'évolution du sentiment d'appartenance de la population qui est étudiée à travers les grands événements de la vie des œuvres, de la conception du projet à aujourd'hui.

LAVOIE, Richard. La Chasse à la sauvagine au-delà du plomb et du sang. Mémoire (M. A.), Université Laval, 1998, xxi-259 p. ill., cartes. [Direction : Jean Simard].

Le contenu de ce mémoire porte sur le phénomène de la chasse à la sauvagine dans la partie haute de l'estuaire moyen du fleuve Saint-Laurent de même que chez les Cris de la Baie James et les Montagnais de la Côte Nord. Le périmètre de la recherche concerne principalement ce que l'on pourrait appeler le couloir migratoire traditionnel de la grande oie des neiges au Québec, c'est-à-dire les régions situées entre Saint-Vallier et Cap-Saint-Ignace, sur la côte sud du fleuve Saint-Laurent, et entre Beauport et le cap Tourmente, du côté nord. L'on y présente l'écologie des rapports d'interaction entre les humains et la sauvagine, les principales espèces de sauvagine chassées, l'ethnohistoire de la chasse à la sauvagine de même qu'une ethnographie des pratiques contemporaines de chasse ainsi que quelques activités limitrophes.

LI, SHENwEN. Stratégies missionnaires des jésuites français en NouvelleFrance et en Chine au XVII siècle. Thèse (Ph. D.), Université Laval, 1998, xiii-344 p. ill., cartes. [Direction : Laurier Turgeon ; codirection : Denys Delâge]. [Thèse publiée sous le même titre : [Montréal], L'Harmattan, «Interculture », 2001, XVI-379 p.]. 
Les jésuites français œuvrent simultanément en Amérique du Nord et en Chine au XVII ${ }^{\mathrm{e}}$ siècle et y développent des stratégies pour essayer de convertir les autochtones. De ce fait, ils y jouent un rôle d'intermédiaire dans les échanges culturels Amérique-Europe et Chine-Occident. Notre étude comparée porte d'abord sur les actions missionnaires et ensuite sur les réactions des Amérindiens et des Chinois. Plus précisément, nous essayons de saisir la variété et la parenté des politiques apostoliques des jésuites français dans les deux milieux culturels d'accueil, de même que l'attitude des deux populations autochtones face à l'expansion du christianisme. Nous étudions l'adaptation des missionnaires à ces deux peuples en vue de cerner les phénomènes de rencontres interculturelles et d'acculturation et de favoriser la compréhension des dialogues et des échanges entre la culture d'origine européenne et les cultures amérindienne et chinoise. Cette étude s'appuie sur une base documentaire large et variée que nous avons construite lors de dépouillements faits au Canada, en France et en Chine.

MARCotTe, Christian. Les Relations entre les trappeurs et les animaux à fourrure : naissance d'une nouvelle génération. Mémoire (M. A.), Université Laval, 2000, vii-155 p. ill., cartes. [Direction : Marcel Moussette].

Basé à la fois sur six enquêtes orales menées sur le terrain et sur trois sources éditées, ce mémoire propose un regard diachronique sur les relations qui unissent les trappeurs de la région de Baie-Comeau aux animaux à fourrure qu'ils recherchent. Pour arriver à ce résultat final, nous devrons parcourir successivement les trois chapitres de cette étude qui se présentent comme suit : un portrait sociogéographique de la région comportant la géographie, les animaux et un historique de la ville de BaieComeau et de ses environs ; une description de la méthodologie utilisée pour la cueillette des données et leur analyse basée sur un système de onze attitudes fondamentales envers les animaux; et finalement, le corps du mémoire, l'analyse, c'est-à-dire, les résultats de l'enquête auprès des auteurs et des informateurs.

Ndoye, Amadou. Les Relations interculturelles entre les immigrants d'origine sénégalaise et la population d'accueil québécoise : jalons d'une analyse des systèmes de représentation et des stratégies d'intégration. Thèse ( $\mathrm{Ph} . \mathrm{D}$.), Université Laval, 1999, xv-388 p. [Direction : Lucille Guilbert].

Cette thèse s'intéresse particulièrement à la problématique interculturelle. En effet, nous avons, dans une dynamique interpersonnelle et relationnelle, cerné les différents systèmes de représentation et les stratégies d'intégration des immigrants d'origine sénégalaise vivant au Québec. Plus précisément, pour mieux comprendre les enjeux des relations 
interculturelles entre Sénégalais et Québécois, notre perspective théorique d'approche tient fondamentalement compte de la structure identitaire traditionnelle de la culture d'origine - soit vers l'identification au groupe (les Sénégalais), soit vers l'autonomie de l'individu (les Québécois de souche). À l'aide de techniques d'enquête, d'entrevues semi-directives en profondeur et de méthodes qualitatives d'analyse de contenu du discours sémiotique, nos conclusions démontrent que l'héritage culturel contribue à influer sur les perceptions identitaires, les stratégies d'intégration des Sénégalais dans la société pluraliste d'accueil québécoise, et que ces derniers, en tant qu'acteurs sociaux inscrits dans des dynamiques d'immigration et relationnelles variantes, ont aussi des façons individuelles de répondre aux normes du groupe.

Parcoret, Florence. L'Amertune des Gardiens de la Terre : les Memekueshuat dans la tradition orale innue. Mémoire (M. A.), Université Laval, 2000, viii116-[6] p. ill., carte. [Direction: Daniel Arsenault ; codirection : Laurier Turgeon].

Ce mémoire constitue un exercice exploratoire sur le thème des Memekueshuat dans la tradition orale des Innus de la Haute et de la Basse Côte-Nord du Québec. Il traite du genre de relation établi entre les Innus et les Memekueshuat et examine plus particulièrement comment la rencontre avec ces derniers fut interprétée, comprise et actualisée au cours des générations par les Innus. Il montre que les Memekueshuat faisaient entièrement partie de l'univers spirituel des Innus, qu'ils avaient leur place au sein du cosmos et qu'ils étaient des êtres avec lesquels les Innus établirent des liens étroits et privilégiés. Ainsi, ce mémoire veut rendre compte de la manière dont les Innus vivaient et concevaient la croyance aux Memekueshuat dans leur environnement, et apporter un éclairage supplémentaire sur leur système de pensée et leur vision du monde.

PICHETTE, MARIE-HÉLĖNE. Musique populaire et identité franco-ontariennes. La Nuit sur l'étang. Mémoire (M. A.), Université d'Ottawa, 2000, [1]-vi160 p. ill. [Direction: Jocelyne Guilbault]. [Mémoire publié sous le même titre : Sudbury, Prise de parole/Société historique du Nouvel-Ontario, 2001, 130 p.]

Ce mémoire examine la construction de l'identité à travers un événement musical annuel, la Nuit sur l'étang, qui a été institué à Sudbury (Ontario) en 1973. Par l'analyse des sources disponibles, il recherche les éléments qui s'avèrent essentiels à la formulation de l'identité franco-ontarienne. Après avoir choisi trois dates charnières - la fondation en 1973, la dixième Nuit en 1983 et la vingt-cinquième en 1998 - et mis en contexte ces 
manifestations, l'étude scrute les discours de la presse et des témoins de l'époque de même que le contenu du spectacle lui-même. Apparaissent alors les composantes qui ont servi à l'élaboration de cette identité. D'une part, l'analyse tend alors à démontrer que l'identité n'est pas un fait acquis, mais que, tout au contraire, elle se construit à travers les éléments et les événements qui la forment. D'autre part, elle illustre comment musique populaire et identité franco-ontariennes se définissent et s'influencent mutuellement dans le cadre de la Nuit sur l'étang.

VALLIÈres, Nicole. La Robe montréalaise bourgeoise, 1870-1883 : clichés, tendances et contextes de consommation. Thèse ( $\mathrm{Ph}$. D.), Université Laval, 1999, xix-437 p. ill. [Direction : Jocelyne Mathieu].

Le vêtement s'inscrit dans une dynamique précise, répondant en cela à des questions qui se rapportent aux conditions et aux modes de vie de ses usagers, c'est-à-dire l'univers domestique. Inscrivant notre recherche dans une perspective d'ethnologie historique, nous formons l'hypothèse que le vêtement (la robe) féminin bourgeois de la fin du XIX ${ }^{\mathrm{e}}$ siècle fait partie intégrante d'un système vestimentaire et que, de ce fait, il est le reflet de la culture d'une certaine bourgeoisie. Dans cette optique, nous avons entrepris l'analyse de 236 robes illustrées dans le corpus de photographies du fonds Notman pour en déterminer les tendances stylistiques et morphologiques, les avons confrontées avec celles retrouvées dans les planches de mode des hebdomadaires montréalais L'Opinion publique et le Canadian Illustrated News, et finalement situées dans un contexte plus large de consommation abordé par la lorgnette des annonces publicitaires publiées dans les deux hebdos. 